\title{
Problems in the Diagnosis and Treatment of Pseudotumor Cerebri
}

\author{
James J. Corbett
}

Can. J. Neurol. Sci. 1983: 10:221-229

It is an honor to be asked to deliver the Silversides Lecture. Dr. Silversides' clear understanding of the need for the collaboration of the basic and clinical sciences is a measure of the good sense for which he is admired. For a clinician, the greatest challenge to his clinical judgment lies in formulation of strategies to deal with vexing and serious conditions. Often we are called upon to treat conditions for which no satisfactory pathophysiologic mechanism has been elucidated. It is said that the clinician must frequently "get on top of the problem before he gets to the bottom of it." In the spirit of all clinicians who seek better clinical approaches to poorly understood disease, I will devote this discussion to the diagnosis and treatment of pseudotumor cerebri.

Pseudotumor cerebri is a condition of obscure cause, the characteristics of which are: a) increased intracranial pressure without evidence of a mass lesion or enlargement of the ventricles, b) normal cerebrospinal fluid cytology and chemistry, c) headache, blurring of vision, transient visual obscurations, diplopia and swollen optic discs in an otherwise well person, d) normal mental status, and e) though this constellation occurs in both sexes, from childhood in the seventh decade, most patients are obese women of childbearing age.

Pseudotumor cerebri is a diagnosis which has come to have a multitude of associations, some of which have been reputed to be causal. Pseudotumor due to Vitamin A intoxication, tetracyclines, steroid withdrawal, or intracranial venous sinus occlusion, is commonly seen in children or adolescents and uncommonly found in adults. Conditions such as menstrual irregularities, pregnancy, birth control pill use, or iron deficiency anemia, are all common conditions of women. Most of these relationships are probably better thought of as chance associations. If patients with systemic lupus erythematosus, vitamin A overdose, renal failure, steroid withdrawal, Cushing's disease, trauma and venous sinus occlusions are excluded, the incidence of idiopathic pseudotumor cerebri in women greatly outweighs men. This has certainly been our experience at the University of Iowa over the last 5 years.

Now that we have said what pseudotumor is, we should say what it is not. Bucheit et al (1969) listed an enormous number of conditions reported with the basic constellation of increased spinal fluid pressure, accompanied by papilledema but no focal neurologic signs. Today virtually all of these conditions can be eliminated with the use of CT scanning, cerebral angiography, and lumbar puncture. Under no circumstances should the diagnosis of pseudotumor be made without finding elevated spinal fluid pressure on lumbar puncture, nor should it be made in the presence of any CSF abnormalities. The following list includes some of the common conditions which have initially been given the diagnosis of pseudotumor at our institution or in recent reports and which probably stand an excellent chance of being mistaken as such again.

1. Tumors

a) Unrecognized gliomas of the brain: This is about $3 \%$ of gliomas which remain isodense on CT with little of no focal signs.

b) Gliomatosis cerebri: while we have not recognized an example of this rare tumor, they enlarge the brain with no focal signs until quite late (Couch and Weiss, 1974).

c) Metastatic tumors from systemic malignancies in the subarachnoid space or subarachnoid seeding by intracranial tumors such as glioblastoma multiforme, medulloblastoma or lymphoma. Cord tumors may also give increased CSF presure and papilledema (Ridsdale and Moseley, 1978). Strangely enough these tumors did not produce hydrocephalus.

2. Arteriovenous malformation (AVM): Though not usually missed on CT scan, there are a number of case reports (Spallone, 1981; Vassilouthis, 1979) which reflect the occasional difficulty this diagnosis may provide. (fig. 1)

3. Infectious diseases:

a. Viral encephalitis may be chronic and indolent and may occur without fever. In these instances the CSF is diagnostic (figure 2).

b. CNS syphilis with optic papillitis may occur with disc swelling and little visual loss. (figure 3 ).

4. The commonest cause of a mistaken diagnosis of pseudotumor cerebri is the combination of optic disc drusen or an anomalously elevated disc and headache. This combination in an obese young woman who also has borderline normal CSF pressure (between 200-250 $\mathrm{mm} \mathrm{H}_{2}$ ) guarantees confusion and may result in long term treatment for non-disease. Transient visual obscurations (Lorentzen, 1966) and hemorrhages (fig. 4) on the disc (Hitchings et al, 1976) and visual field defects are all commonly seen with drusen. Fluorescein angiography may help to clarify the diagnosis. While occasionally normal in mild papilledema, fluorescein usually leaks diffusely with

This paper was presented as the 2nd annual J.L. Silversides Lecture, at the Toronto Western Hospital, and the Academy of Medicine. Toronto. June 18. 1982. Reprint requests to: James J. Corbett, M.D.. Associate Professor, Department of Neurology, University Hospitals, Iowa City, lowa 52242 


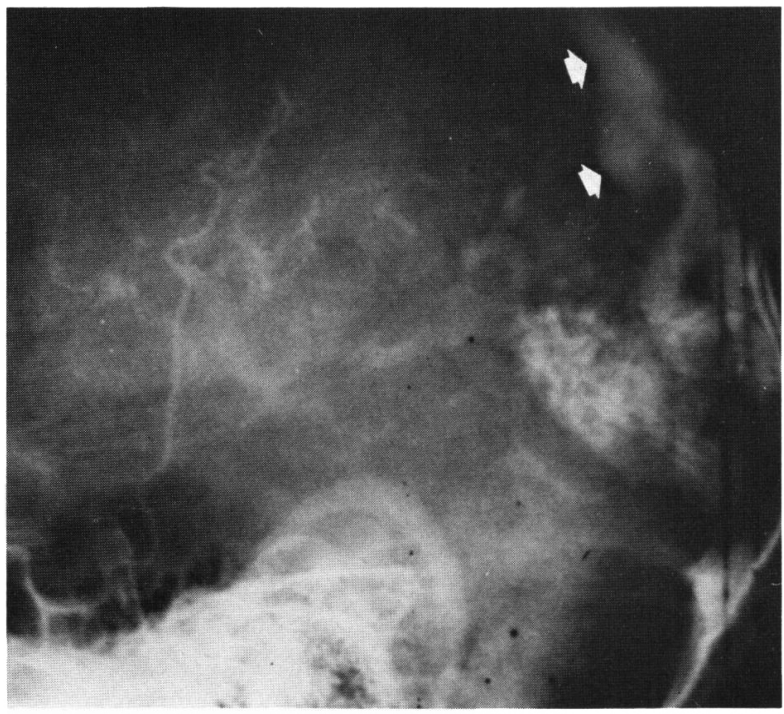

Figure I - An AVM in a man with headaches, papilledema, horizontal diplopia and no focal neurologic signs seen before the CT era. The major draining vein (arrows) was directed anteriorly into the superior sagittal sinus. Papilledema disappeared with removal of the AVM.

Figure 2 - Bilateral papilledema due to encephalitis. This woman had chronic headache, transient visual obscurations and horizontal diplopia for over a month. The diagnosis of encephalitis was made by lumbar puncture where the white count was elevated, CSF protein was elevated with $75 \%$ gamma globulin and elevated serum adenovirus titers despite absence of fever. This disc swelling is indistinguishable from any other disc swelling.
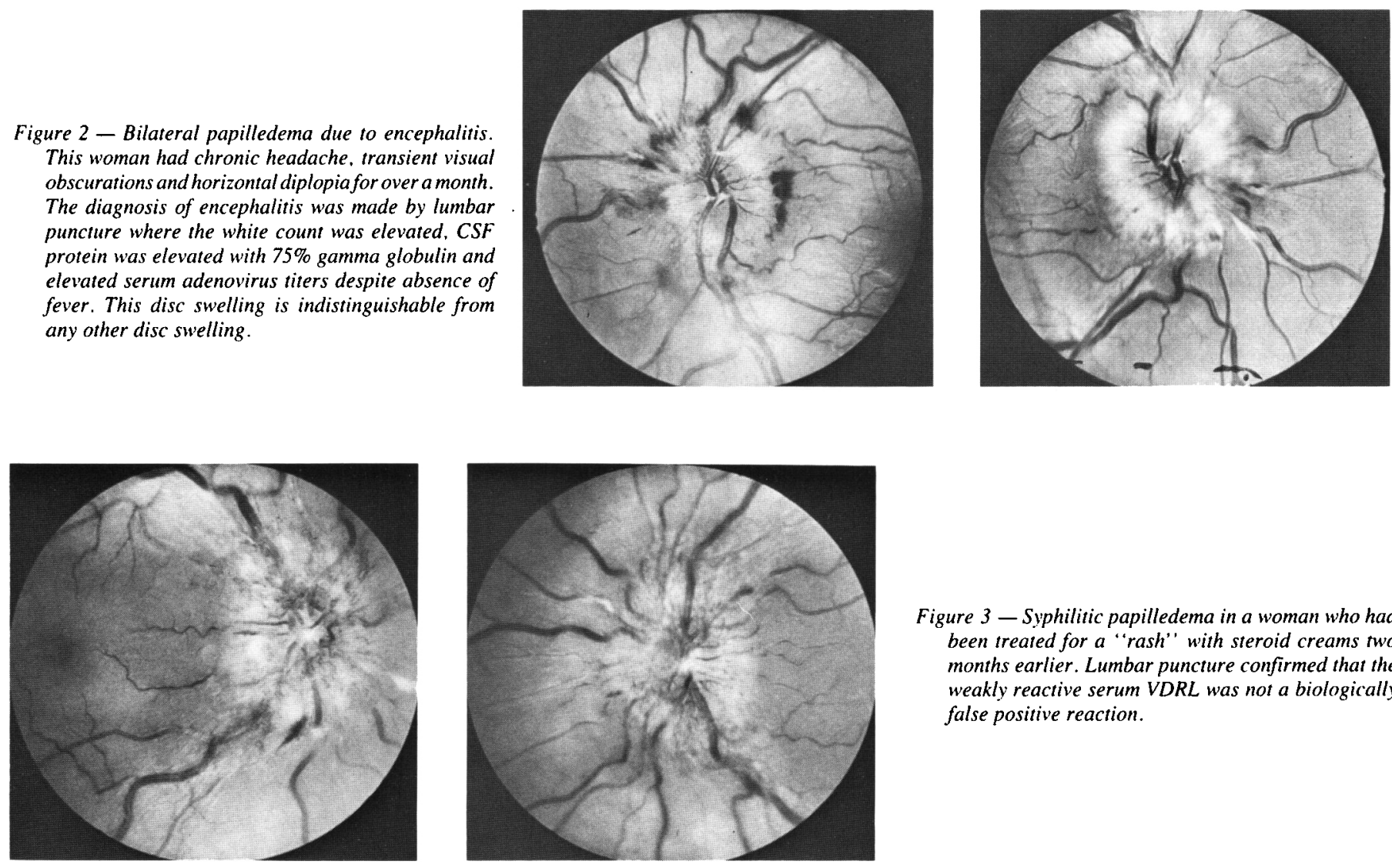

Figure 3 - Syphilitic papilledema in a woman who had been treated for a "rash" with steroid creams two months earlier. Lumbar puncture confirmed that the weakly reactive serum VDRL was not a biologically false positive reaction.

papilledema and leaves hot-spots of late focal fluorescence with drusen. $25 \%$ of a group of patients reported by Johnston and Paterson (1974) had normal CSF pressure on prolonged recording and negative fluorescein angiography suggesting that they had pseudopapilledema.

\section{SYMPTOMS AND SignS}

The symptoms of pseudotumor, aside from headache, are mainly visual. Monocular and binocular transient visual obscura- tion (TVOs) are seconds-long blackouts of vision probably due to hypotension in the visual pathway caused by transient changes in intracranial pressure. While many of these TVO occur with postural change, they may occur while sitting quietly. Transient visual obscurations are not predictive of permanent visual loss (Rush, 1980; Corbett et al, 1982) and TVO may disappear with or without reduction of CSF pressure. Transient visual obscurations may also be seen with myopia, and in $40 \%$ of patients with optic disc drusen (Lorentzen, 1966). This provides further confusion in the differentiation between papilledema and pseudopapilledema. 


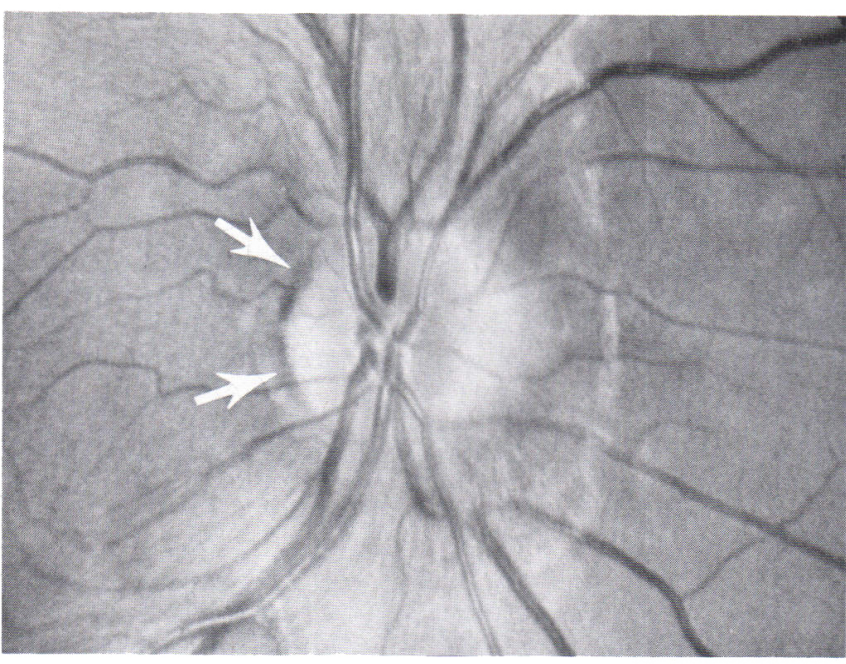

Figure 4 - Typical hemorrhage seen with optic nerve head drusen. (arrows)

Diplopia is almost always horizontal, rarely vertical, and is due to the non-specific, false localizing effect of increased pressure on the abducens nerve. The rare reports of skew deviation, (Merikangas, 1978), IVth nerve (Halpern and Gordon, 1981) and IIIrd nerve paresis (Grant, 1981; Snyder and Frenkel, 1978; McCammon et al, 1981) should not make one comfortable about the diagnosis of pseudotumor. Patients with diplopia due to causes other than abducens nerve paresis require a closer look for other causes of increased CSF pressure.

Headache is an initial symptom in virtually all patients first seen by neurologists but may be absent in 10-20\% or more of those who first seek medical attention from an ophthalmologist because of transient visual obscurations, diplopia or visual loss (Rush, 1980; Corbett et al., 1982). There is nothing specific about these headaches which may be global or one-sided, mild or severe. Pre-existing migraine may worsen with pseudotumor.

Very rarely patients will have severe aching in the neck, arms, and back, as well as headache, as an initial complaint. These patients may even have meningismus. Widely dilated spinal root sleeves were demonstrated with radionuclide scanning in one report (Bortoluzzi et al, 1982). These authors suggested that it is the dilated root sleeves with increased pressure on the spinal roots, which causes such discomfort.
Occasionally a patient will complain of an intracranial bruit with noise in one ear or both. It is probably prudent to consider the possibility of venous sinus occlusion or arteriovenous malformation in these cases and perform arteriography with emphasis on the late venous phases or digital venous subtraction angiography.

Facial paresis (Chutorian et al, 1977) and facial pain (Hart and Carter, 1982) have been reported in occasional cases of pseudotumor. These features are sufficiently unusual to warrant studies beyond CT scan to rule out tumor, venous sinus occlusion or arteriovenous malformation. Occasional patients are totally symptom free and are discovered fortuitously during a routine ophthalmologic examination.

Papilledema encountered in pseudotumor cerebri does not appear different from papilledema in any other condition with increased CSF pressure. Splinter or subretinal hemorrhages may be seen around the disc and rarely away from the disc (Keane, 1981;Galvin and Sanders, 1980). Exudates, both superficial fluffy nerve fiber layer infarcts (soft exudates) and deeper, sharper, "hard" exudates are seen. Predominantly unilateral or strictly unilateral disc swelling is occasionally seen. (Kirkham et al, 1973). Patients with longstanding papilledema may develop pale, flat discs which can be particularly confusing if the patient presents with intermittent visual symptoms and elevated spinal fluid pressure.

Complications of high pressure in the optic nerve sheath include chorioretinal folds (Bird and Sanders, 1973) (figure 6) and subretinal hemorrhage (Jamison, 1978; Troost et al, 1979. Morse et al, 1971) (figure 7). Optociliary collateral vessels may appear (Eggers and Sanders, 1980) and disappear (Perlmutter et al, 1980) as the sheath pressure, and resistance to central retinal venous outflow, fluctuate. Macular edema has been reported (Morris and Sanders, 1981) as have nerve fiber layer defects, which provide an objective correlate of visual field loss (figure 8).

It is not possible to be certain how long papilledema has been present by appearance alone. Descriptions should be as "timefree" as possible. Classifications which mix temporal terminology (early, chronic) and novel descriptive words (vintage) imply that the duration of the swelling is known. Since it is rare that we know when the papilledema actually began, it is probably better to speak of mild, moderate, severe and atrophic forms of papilledema, which purely grade severity on the basis of appearance. Frisen (1982) has recommended a classification scheme
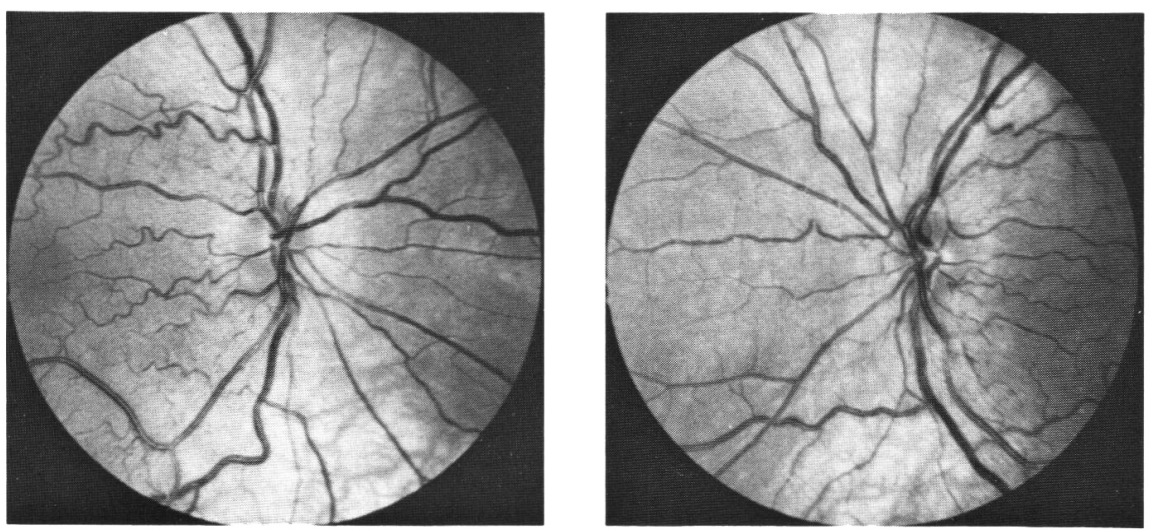

Figure 5 - The "little red disc" is a particularlytroublesome mimic of papilledema. Gowers (1882) pointed out that small discs tend to be hyperemic in appearance and large discs frequently look pale. 

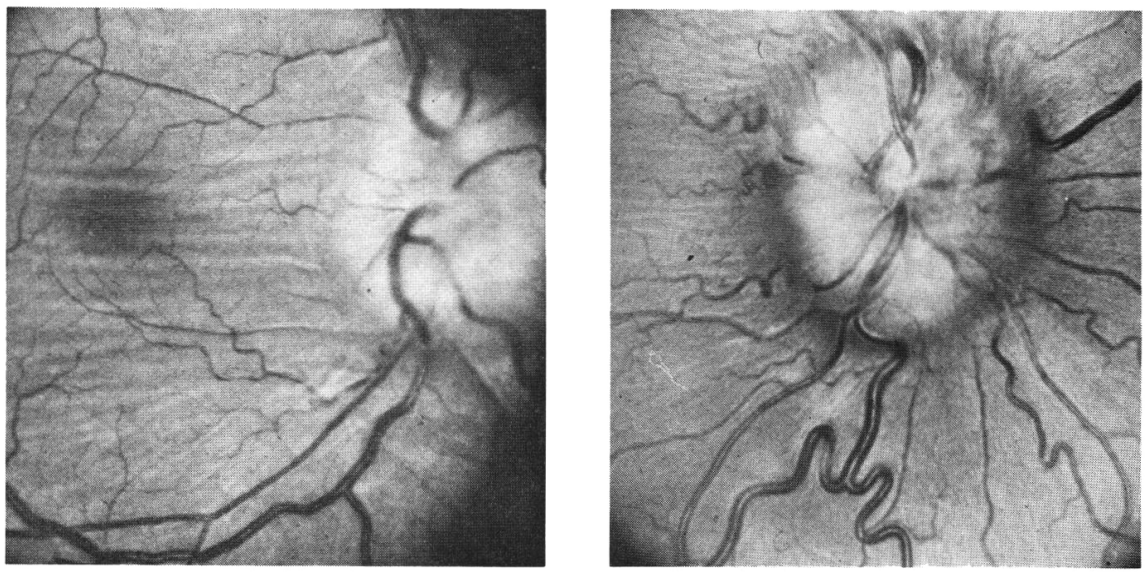

Figure 6 - Two examples of chorioretinal folds in papilledema associated with very large shallow enlargement of the blind spots. These enlarged blind spots remain even after papilledema has disappeared.

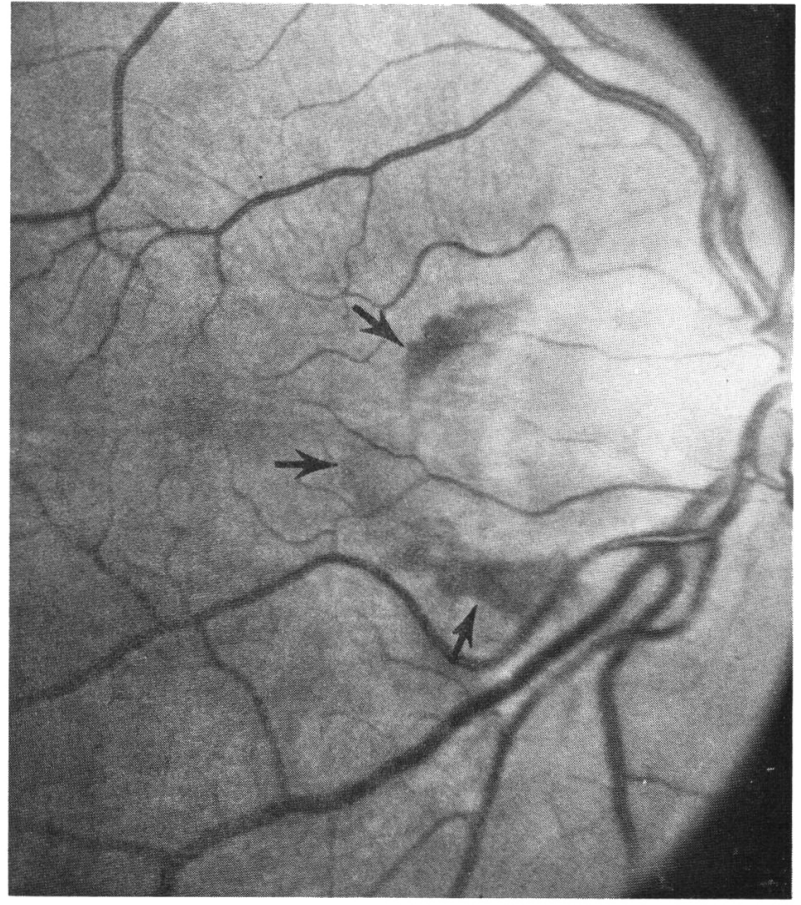

which uses nerve fiber layer opacification, obliteration of vessels, and loss of cup as criteria of severity. Reports of patients with elevated spinal fluid pressure and no papilledema (Spence et al, 1980) may actually be patients whose papilledema has recovered from an acute attack of pseudotumor and have chronic intracranial pressure elevation with resolved papilledema. In half of all patients with pseudotumor papilledema resolves with little or no visible residue of disc swelling and no visual field defect (Corbett et al, 1982).

\section{Visual field disturbance in pseudotumor}

Some degree of visual impairment in the form of visual field defects or depressed visual acuity will occur in about half of patients. We found serious unilateral or bilateral visual loss in about $25 \%$ (Corbett et al, 1982). It is generally agreed that visual loss is the only serious complication of this condition. Enlarged blind spots are found in virtually all patients with papilledema. This has been attributed to displacement of the peripapillary

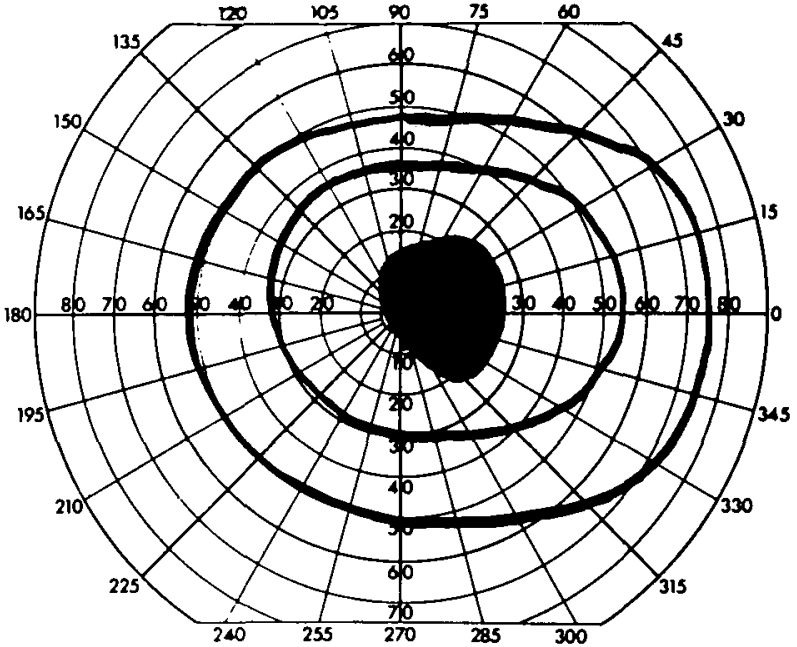

Figure 7 - Subretinal neovascular membrane hemorrhage (arrows) 18 years after a successful subtemporal decompression for pseudotumor. Vision went to $20 / 200$ in this eve.

rods and cones by the swollen axons (Huber, 1971). If there are, in addition, choroidal folds, the blind spot may be enormous (Frisen and Holm, 1978). It may continue to be enlarged long after the papilledema has disappeared if the choroidal folds persist. This suggested to us that the enlarged blind spot, which is a relative, sloping scotoma, was a refractive scotoma, i.e. one which disappears with refraction. We tested this idea by using progressively increasing powers of plus lenses and found that the enlarged blind spot of papilledema systematically shrank. (figure 9). Because blind spot enlargement may persist after the discs flatten, and because other conditions, mimicking papilledema, such as drusen, can also enlarge the blind spot, plotting the blind spot is both an unreliable measure of therapeutic success, and an inaccurate way of following the patient's visual function.

Constriction of the visual field is a less common consequence of chronic papilledema. This may begin with small or shallow arcuate scotomas, and may progress to a dense, nasal, inferior 


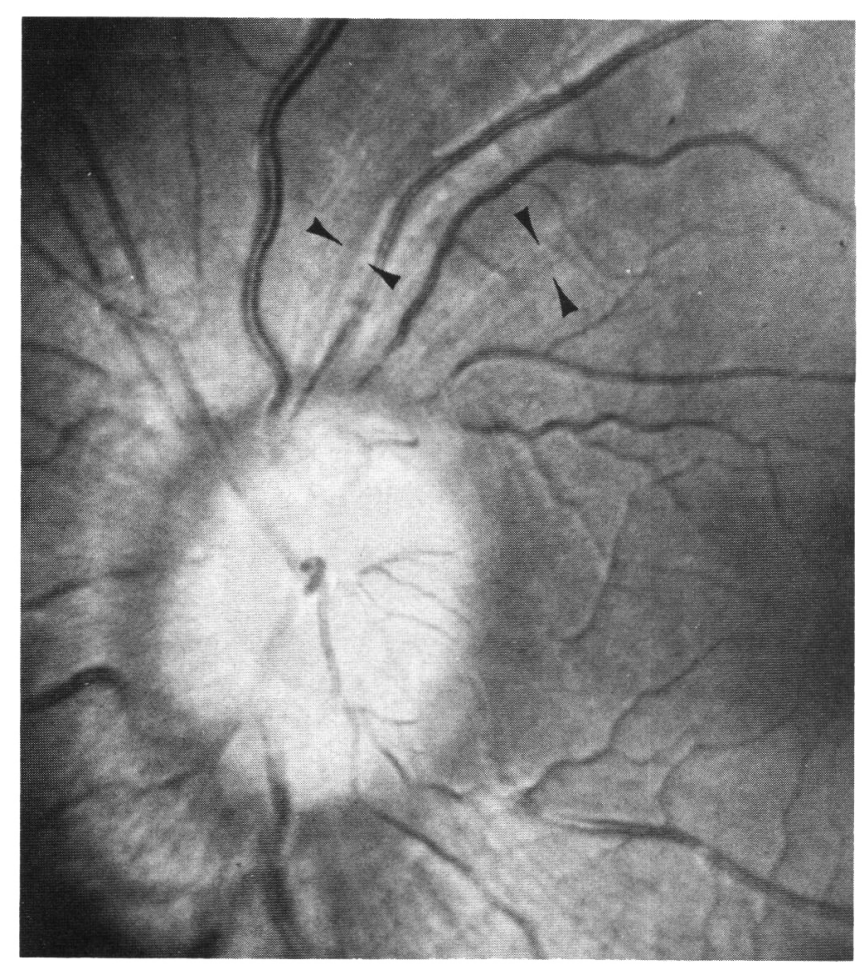

Figure 8 - Nerve fiber layer defects (arrows) clearly seen in a patient with longstanding elevation of the optic disc.

(Dersh and Schlezinger, 1959) or superior visual field defects similar to the nasal step seen in glaucoma. Constriction of the temporal field, usually seen as a pie-shaped defect pointing to the disc may occur but is less common and is more typical of an anomalous optic nerve or hypoplastic disc (Buchanan and Hoyt, 1981).

Central visual field loss may occur following a subretinal hemorrhage in the papillo-macularbundle(Jamison, 1978; Troost

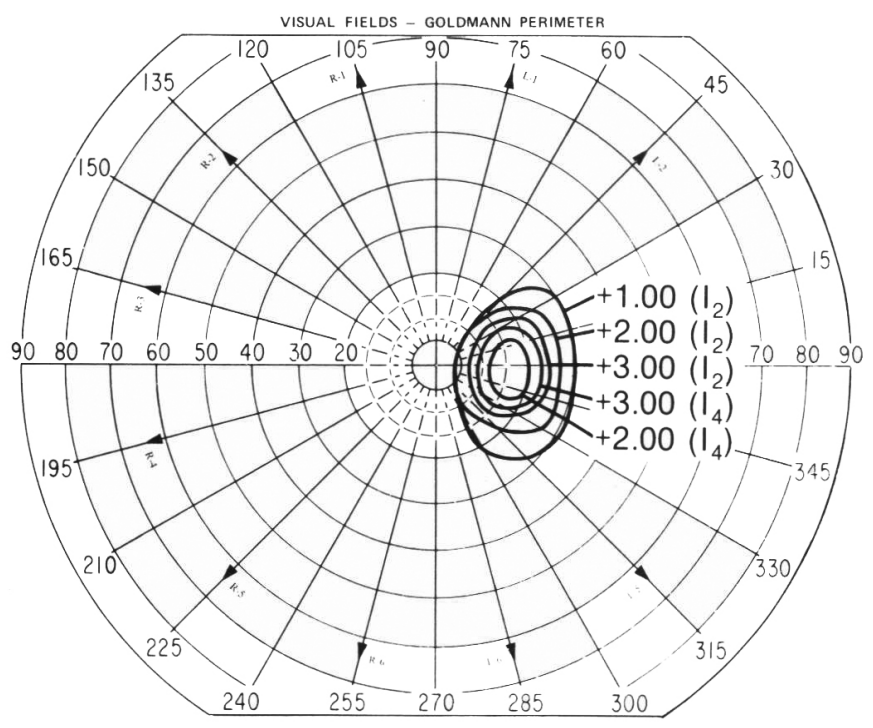

Figure 9-Enlarged blind spot in patient with pseudotumor and a large optic disc. This scotoma shrinks as more plus lens is used. In normal individuals plus lenses nasally displace the blind spot a small amount and trivially minify the blind spot. et al, 1979; Morse et al, 1971) and is more often seen in patients with choroidal folds (Griberg and Grove, 1980). Ischemic damage both due to venous and arteriolar compromise can cause disc infarcts which may contribute to central visual loss (Morris et al, 1970). Frank ischemic optic neuropathy alone (Green et al, 1980), or combined with central retinal vein occlusion (Corbett et al, 1982), is rare, and may cause disastrous visual loss.

\section{Cerebrospinal fluid pressure in pseudotumor cerebri}

Recovery from pseudotumor cerebri is gauged as resolved papilledema and is usually thought to be synonymous with the return of CSF pressure to normal. Foley (1977) reported 6 patients followed for pseudotumor over a number of years who had persistently elevated CSF pressure, which led her to suggest that pseudotumor may be a chronic condition. We subsequently reported 13 patients who underwent lumbar puncture 5 to 41 years after their initial bout of pseudotumor (Corbett et al, 1982), of whom $80 \%$ had pressures of $200 \mathrm{~mm} \mathrm{H}_{2} \mathrm{O}$ or more.

Of particular interest in our group of chronic pseudotumor patients (Corbett and Mehta. 1983, in press) was one man who was initially found to have CSF pressure of $150 \mathrm{~mm} \mathrm{H}_{2} \mathrm{O}$ years post attack: a repeat spinal tap three weeks later showed CSF pressure of $330 \mathrm{~mm} \mathrm{H}_{2} \mathrm{O}$. This is consistent with some of Johnston and Paterson's (1974) observations of wide fluctuations of CSF pressure in patients with pseudotumor.

Just what is "normal" CSF pressure? We have studied CSF pressure in neurologically normal obese and non-obese patients who were undergoing spinal anesthesia (Corbett and Mehta, 1983 in press) and found that the upper level of normal CSF pressure ranged between 200 and $250 \mathrm{~mm} \mathrm{H} \mathrm{H}_{2} \mathrm{O}$ (figure 10). We measured CSF pressure after it had stabilized for one minute rather than waiting for the pressure to drop to some preordained "normal level" (possibly due to leakage). Our findings were consistent with earlier studies performed on normal paid volunteers (Gilland et al, 1974). This question of "normal" pressure needs to be taken into account when measuring CSF pressure in a patient whose discs may be anomalously elevated. Repeat spinal fluid pressure measurement may be required to see whether the CSF pressure is indeed "normal", that is, less than 200 or more than $250 \mathrm{~mm} \mathrm{H}_{2} \mathrm{O}$.

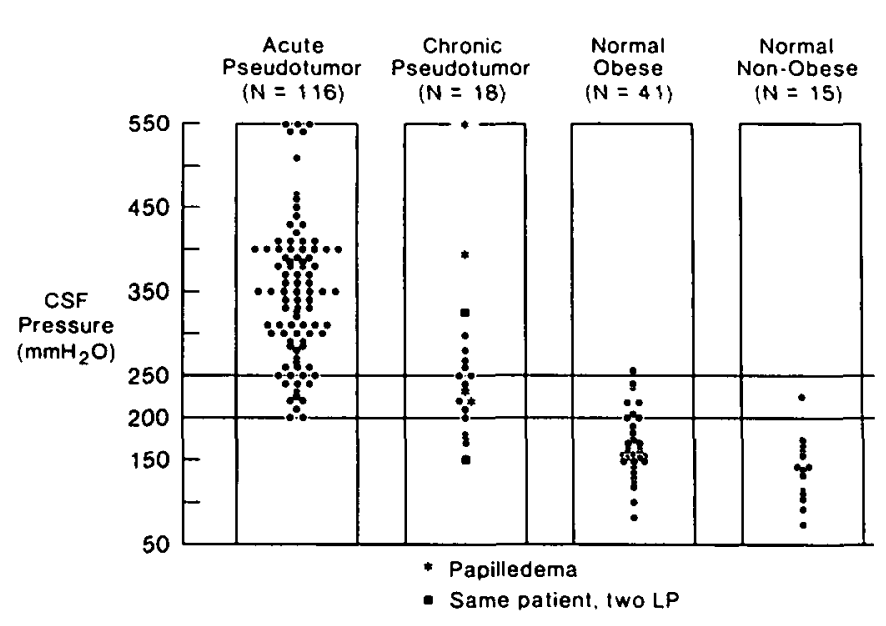

Figure $10-$ CSF pressure in normal obese and non-obese patients and patients with acute and chronic pseudotumor (from Corbett and Mehta. Neurology, 1983). 
Should we avoid measurement of the CSF pressure in any patient? One young woman we studied who had occlusion of the straight sinus developed three episodes of decerebrate posturing with transient brain stem neurologic deficit. These were precipitated by sitting upright 24 to 36 hours following a spinal tap. In such situations lumbar puncture is best avoided, especially if the ambient cisterns are effaced on CT. It is otherwise rare to have a serious complication from lumbar puncture in a patient with idiopathic pseudotumor.

How often is CSF pressure reduced after treatment? Commonly a new, lower equilibrium of CSF pressure is reached though this level is often in the upper range of normal (200-250 $\mathrm{mm} \mathrm{H}_{2} \mathrm{O}$ ) if not mildly elevated. Cooper et al (1979) reported normal resting CSF pressures in 5 of 9 patients with pseudotumor. Normal CSF pressure (clearly less than $200 \mathrm{mg} \mathrm{H}_{2} \mathrm{O}$ ) in a symptomatic patient with pseudotumor who has papilledema is extremely rare in our experience. Normal CSF pressure is more likely to be due to leakage from a multiply fenestrated lumbar sac prior to measurement of CSF pressure.

\section{Which patient should the clinician be uncomfortable about?}

1. Men, regardless of age or body habitus, are so much less likely to have idiopathic pseudotumor that they deserve special scrutiny. One must be careful to exclude those causes of "secondary" pseudotumor mentioned earlier, particularly intracranial venous sinus occlusions due to metastatic malignancies such as prostatic carcinoma and hypernephroma.

2. Any patient who has unusual features of the disease: neck pain, objective or subjective bruits, IIIrd, IVth, Vth, or VIIth nerve involvement.

3. Anyone with focal neurologic signs.

4. Any patient with an abnormal CSF examination: normal pressure, high protein, low glucose or excessive numbers of white cells.

5. Anyone with large ventricles on CT scan.

\section{What other studies should be done?}

After the diagnosis is established it is the visual system which needs to be followed. Careful monitoring of visual fields as if treating the patient for glaucoma, measurement of visual acuity and repeat fundus photographs are the keystones of treatment decisions. An ophthalmologic examination with measurement of intraocular pressure is necessary and should be repeated at regular intervals if steroid medication is used. The ophthalmologist is at least as important as the neurologist in the evaluation and management of any patient with pseudotumor. They are especially important in surgical decision making. Since visual disturbances are the only significant residues of this condition careful attention must be directed to the patients' visual function.

Extensive studies of endocrine function are not necessary, and have proven unrevealing (Reid and Jamison. 1980: Corbett et al, 1982). Abnormalities of calcium metabolism (hypo- or hyperparathyroidism) are the only potentially occult forms of endocrine disease associated with pseudotumor. Those patients with Addison's disease or Cushing's disease who have pseudotumor are not usually difficult diagnostic problems.

Systemic hypertension may contribute to optic disc damage and the visual morbidity of a patient with pseudotumor (Corbett et al, 1982). Though hypertension is treated, rapid or drastic lowering of severely high blood pressure may contribute to ischemic damage to the optic disc in the face of high optic nerve sheath pressure. This must be remembered in patients who have pseudotumor cerebri and are undergoing hemodialysis for chronic renal failure, where hypotensive episodes are common.

Once the diagnosis is established and all the studies confirm a diagnosis of pseudotumor, how do we treat the patient?

There are no randomized prospective studies of therapy for pseudotumor. Recent articles (Weisberg, 1975; Ahlskog and O'Neill, 1982), which review the spectrum of treatments in pseudotumor, speak from the standpoint of chart reviews, and their therapeutic recommendations must therefore be considered weak and tentative. There have been, unfortunately, no studies of the natural history of untreated pseudotumor cerebri. Since the average duration of symptoms in pseudotumor is commonly brief it is difficult to know whether reported improvements are the result of medicine or simply represent the natural course of the illness. Furthermore, symptoms commonly improve on any treatment regimen with no change in disc swelling or CSF pressure. In the imperfect world of the clinic, wanting in scientific precision, what medical and surgical choices do we have?

No treatment, an option rarely considered, is reasonable for the asymptomatic patient who has no visual field loss and normal visual acuity. The reasons to treat are: a drop in visual acuity, loss of visual field, persistent diplopia, or headache. If the patient has none of these, no treatment is needed. In Johnston and Paterson's (1972) series of 75 patients, 11 were given no treatment and all of these recovered spontaneously.

Repeated lumbar puncture has little to recommend it except for relief of severe headache. Even in the best situations a lumbar puncture can be difficult to perform in an obese patient and may, after many taps, contribute to chronic low back pain. Lumbar puncture has only a short lived effect on CSF pressure: Johnston and Paterson (1974) found a return of pressure to pre-tap level after only 82 minutes. A theoretical objection to repeat spinal taps is the risk of developing intraspinal epidermoid tumors presumably caused by implantation of epidermal cells (Batnitzky et al, 1977). The tumor may appear $1 \frac{1}{2}$ to 23 years following the tap. Manno et al (1962) believe that an alarming $41 \%$ of intraspinal epidermoid tumors in their series could be traced to antecedent lumbar punctures.

Because all but about $10 \%$ of pseudotumor patients are obese, weight loss has been advanced as an effective treatment. (Newborg, 1974). Presumably any low calorie diet will work, but any diet is difficult to enforce. Occasional patients will cooperate and benefit from this form of treatment, but one must be careful not to coerce the patient to diet with threats of blindness.

Carbonic anhydrase inhibitors, usually acetazolamide, have long been used to reduce CSF production in patients with hydrocephalus (McCarthy and Reed, 1974; Donat, 1980). Although these drugs have been said to be ineffective on a chronic basis, the dosages recommended have been relatively small. The one exception has been the report of Gücer and Vierenstein (1978), who recommended doses four to eight times the usual dose of $250 \mathrm{mg}$ twice daily. We find it preferrable to use the $500 \mathrm{mg}$ capsules twice daily rather than three or four doses of $250 \mathrm{mg}$.

The most effective dose is as yet undetermined. Side effects are dose related and include: commonly tingling in the fingers, toes, and perioral region, and less commonly nausea, anorexia, and distaste for carbonated beverages. Rarely patients will develop renal stones. Metabolic acidosis, evidenced by lowered serum bicarbonate, is a good measure of compliance. 
Thiazide diuretics, furosemide, and ethacrynic acid (McCarthy and Reed, 1974) have all been used to treat pseudotumor but a good prospective randomized trial is not to be found. No single diuretic has been proven clearly superior to any other. Jefferson and Clark (1976) found that chlorthalidone, a long acting thiazide, was effective, but it is unclear whether the results they reported represent a drug effect or the illness's natural history. Large doses of ethacrynic acid and furosemide often necessitate frequent urination, which may be a problem for the patient at work or during sleep.

With the use of corticosteroids, we come to the "strongmedicine-for-serious-problems" school of therapeutics. Although steroids are effective in treating pseudotumor, either alone or in combination with other drugs (Johnston and Paterson, 1974), recurrence of papilledema is common following tapering, and the physician is tempted to return to the use of higher doses of corticosteroids for progressively longer courses of treatment. Curiously, CSF pressure often remains elevated on corticosteroids even if papilledema disappears (Johnston and Paterson, 1972). Side effects such as acne, hair loss, greater weight gain, and dermal stria are serious problems to obese women already doubtful about their appearance. Systemic hypertension and increased intraocular pressure can be produced with corticosteroids (Corbett et al, 1982) and may further aggravate visual loss. Our personal experience with this burst and taper technique has been disappointing.

Oral glycerol is a form of cerebral dehydration first recommended in 1963 to reduce intracranial pressure. Glycerol is made more palatable by mixing with ice and lemon flavoring, but nausea remains the major limiting side effect. A single dose of one gram $/ \mathrm{kg}$ of glycerol will raise serum osmolality from 295 to $320 \mathrm{mOsm} / \mathrm{L}$ in 90 minutes, and reduce CSF pressure for 3 to 5 hours. Doses given every four hours can result in a reversed osmotic gradient and a rebound increase in intracranial pressure (Guisado et al. 1975; Rottenberg et al. 1977) while a six hour interval is too long and allows the pressure to recur. Nevertheless, glycerol may give symptomatic relief from headache and diplopia. Some caution is indicated, however, as hyperosmolar non-ketotic coma has been reported with the use of glycerol (Oakley and Ellis, 1976). Together, the added calories, the large volume of glycerol needed, the awkwardness for a working person to use this medication, the nauseating side effects, and the potential hyperosmolar state all militate against the use of glycerol.

Cardiac glycosides have been recommended by Neblett et al (1972), but they have never been studied seriously in large numbers of pseudotumor patients, and therefore cannot be recommended at this time.

Which medical regimen is best?

Assuming the patient has headaches which do not disappear with the first spinal tap, or continues to have double vision or visual obscurations, virtually any of the treatments mentioned may be used in an attempt to reduce symptoms. One to three acetazolamide capsules a day is a good way to begin treatment, along with a reducing diet. If carbonic anhydrase inhibitors are not tolerated, any other diuretic can be used, paying special attention to hypokalemia. If these medications fail to relieve the symptoms, repeated spinal taps or steroids may be used. In our opinion, long courses of steroids should be avoided for the reasons mentioned above. If steroids are used, intraocular pressure should be measured at one month intervals. An alter- native treatment to be used, before starting a patient on steroids for headaches, is to try the standard migraine drugs such as beta blockers.

How should the patient be followed and how is therapy assessed? Success of therapy is judged by relief of headache, elimination of transient visual obscurations and diplopia, and reduction of papilledema. Repeated fundus photos are far more helpful in accurate assessment of papilledema than descriptions of the optic disc, unless the descriptions are accompanied by meticulous drawings. It is common to find little change in photos despite the clinical ophthalmoscopic impression that the discs were either "improved" or "worse". Stability of, or improvement of, the visual field is evidence of effective treatment: this is the goal of all treatment and it is the most important step in gauging the course of the illness. If the patient is unable to cooperate for reliable visual field examinations, the physician is left to making therapeutic decisions by speculation. Superimposed functional ("hysterical") visual loss can make such decisions vexing (Case \#15, Corbett et al, 1982).

There are no good objective visual tests to measure therapeutic success. Both the objective VER and the subjective color vision tests (both A-O plates or the Farnsworth-Munsell 100 hue test) are less sensitive than quantitative visual field testing for the detection of visual loss. Occasionally, a relative afferent pupillary defect will uncover visual damage before perimetry picks up a field defect. It is possible that static visual fields are more sensitive than kinetic fields in identifying early visual field loss. Recent studies correlating visual field loss with optic nerve axon dropout show that as many as $40 \%$ of axons may be lost before a visual field defect is detectable (Quigley et al, 1982).

CSF pressure may remain elevated in patients for years, even after papilledema is gone. Intermittent sampling of pressure by lumbar puncture is not an effective way of telling whether the pressure is persistently elevated. Though continuous measurement of CSF pressure by ventricular catheters or implanted epidural pressure transducers can monitor the patient's intracranial pressure chronically (Cooper et al, 1979; Johnston and Paterson, 1974; Spence et al, 1980; Gücer and Vierenstein, 1978), such measurement is unnecessary, in that clinical management is guided by visual fields and visual acuity and not by the CSF pressure itself.

Measurement of the degree of disc swelling - using contour disc photography — has been developed by Shapiro (1979). This technique allows measurement of the disc elevation and can be repeated from examination to examination with a high degree of accuracy. While still investigational, this technique may prove to be an important, reproducible, objective measure of treatment success.

Treatment failure is defined as the persistance of headache, diplopia, TVOs, or loss of vision. While papilledema usually disappears within months of the institution of treatment, it may remain for a year or more with varying effects on vision. Incapacitating headache is unusual and recurrent transient visual obscurations or persistent diplopia are all but unheard of.

What should be done if visual field loss continues to worsen despite medical treatment?

Because no single drug or combination of medications has been proven to be more effective than any other drug, in the face of failing vision, one should not delay surgical treatment by trying a number of different drugs. Surgical decompression 
should be done before, not after, serious visual loss has occurred. As most of this loss is peripheral visual field constriction, while visual acuity remains normal, progressive constriction of the visual field is reason enough to turn from medical to surgical therapy. It should be emphasized that there is no "acceptable" level of visual field or acuity loss which one should wait for visual loss which continues despite optimum medical therapy is sufficient reason to turn to decompression.

\section{Surgical Treatment}

Subtemporal decompression which is no longer in the surgical repetoire, was at one time the mainstay of treatment. It was quite effective in circumstances of even profound visual loss (Corbett et al, 1982). The complications of this procedure, namely stroke and seizure (Wilson and Gardner, 1966), were probably related to herniation of brain through the craniotomy site resulting in infarction, and seizures. Incision of the dura during this operation, permitted intimate contact between the arachnoid and the galea, the latter serving as a surface for CSF absorption. The galea absorption mechanism is now used occasionally as a temporary treatment of hydrocephalus with ventriculo-subgaleal shunts. (Perret and Graf, 1977). Though subtemporal decompressions were effective, their high complication rate led to use of alternative surgical shunting procedures.

Lumbar-peritoneal shunting procedures (Vander Ark et al, 1974) are, unfortunately, also frought with problems. The replacement of polyethylene by silastic tubing has eliminated the formerly high rate of post-operative scoliosis and arachnoiditis. While single-piece systems have overcome disconnection problems, they have not circumvented the considerable difficulties of repeatedly testing the function of the system, nor have they eliminated the propensity for the intrathecal end of the tube to become clogged. Other valve problems, such as persitently increased CSF pressure or a low pressure, continue to be troublesome. Though frequently advocated as a surgical treatment, there is as yet no large series with long follow up of patients who have undergone lumbar-peritoneal shunts for pseudotumor. Ventriculo-peritoneal (Johnston and Paterson, 1972) and cisternal-peritoneal shunts (Beatty, 1983) have also been used, but again, no large series of follow up has been reported.

Optic nerve sheath decompression was first done by De Wecker (1897). Interest in the procedure was revived by Hayreh (1964), with his animal work, in which he opened a window of dura in the optic nerve sheath in monkeys who had experimental papilledema produced by an intracranial balloon. The procedure was described as effective in humans by Galbraith and Sullivan in 1973. The intra-orbital portion of the optic nerve is approached by either a medial or lateral orbitotomy. I advocate optic nerve sheath decompression before serious visual loss has ensued. The procedure is effective in eliminating papilledema and rapidly stabilizing or reversing visual acuity and visual loss. The mechanism is not entirely clear, since CSF pressure, as measured by LP, is not lowered (Kaye et al, 1981). However, it is likely that the sheath collapses around the optic nerve and continues to leak small amounts of CSF until the subarachnoid space again closes, as suggested by a histologic investigation in one such patient (Keltner et al, 1977). We have had good success with this procedure.

\section{Conclusions}

The diagnosis of pseudotumor is straightforward if one follows the rule of always doing a lumbar puncture. The combina- tion of lumbar puncture, CT scan, and in appropriate cases, cerebral angiography, should eliminate other illnesses which are confused with pseudotumor. With the diagnosis established, patients should be followed closely until the status of their visual field and visual acuity is clear. An ophthalmologist who has access to fundus photography and Goldmann visual fields should work with the neurologist or neurosurgeon. The patient's spinal fluid pressure is not important in management since the CSF pressure commonly remains elevated after the discs flatten.

Treatment may consist of any number of diuretics, along with a reducing diet. Steroids should not be used for long periods of time, and if used necessitate monitoring of both blood pressure and intraocular pressure at monthly intervals. Should visual loss occur despite medical therapy, optic nerve sheath decompression is recommended. There is no compelling reason to treat asymptomatic patients who do not have loss of visual acuity or visual field.

\section{ACKNOWLEDGEMENT}

This work was supported in part by grant RR59 from the General Clinical Research Center Branch of the NIH.

\section{REFERENCES}

Ahlskog, J.E. and O'Neill, B.P., (1982) Pseudotumor cerebri. Ann. Int. Med. 97: 249-256.

Batnitzky S., et. al., (1977) Iatrogenic intraspinal epidermoid tumors. J. Am. Med. Asso. 237: 148-150.

Beatty, R.A., (1982) Cervical-peritoneal shunt in the treatment of pseudotumor cerebri. J. Neurosurg. 57: 853-855.

Bird, A.C. and Sanders, M.D., (1973) Choroidal folds in association with papilloedema. Br. J. Ophthalmol. 57: 89-97.

Bortoluzzi, M., Di Lauro, L. and Marini, G., (1982) Benign intracranial hypertension with spinal and radicular pain. J. Neurosurg. 57: 833-836.

Buchanan, T.A.S. and Hoyt, W.F., (1981) Temporal visual field defects associated with nasal hypoplasia of the disc. Br. J. Ophthalmol. 65: 636-640.

Buchheit, W.A., et al., (1969) Papilledema and idiopathic intracranial hypertension: Report of familial occurrence. New Eng. J. Med. 280: 938-942.

Chutorian, A.M., et. al., (1977) Benign intracranial hypertension and Bell's palsy. New Eng. J. Med. 296: 1214-1215.

Cooper, P.R., Moody, S., and Sklar, F., (1979) Chronic monitoring of intracranial pressure using an in vivo calibrating sensor: Experience in patients with pseudotumor cerebri. Neurosurg. 5: 666-670.

Corbett, J.J., et. al., (1982) Visual loss in pseudotumor cerebri. Arch Neurol. 39: 461:474.

Corbett, J.J. and Mehta, M.P., (1983) Cerebrospinal fluid pressure in normal obese subjects and patients with pseudotumor cerebri. Neurology.

Couch, J.R. and Weiss, S.A., (1974) Gliomatosis cerebri. Neurology 24:504-511.

Dersh, J. and Schlezinger, N.S., (1959) Inferior nasal quadrantanopsia in pseudotumor cerebri. Arch. Neurol. 1: 695-699.

DeWecker, L., (1872) On incision of the optic nerve in cases of neuroretinitis. Int. Ophthalmol. Congr. Rep. 4: 11-12.

Donat, J.F., (1980) Acetazolamide-induced improvement in hydrocephalus. Arch. Neurol. 37: 376.

Eggers, H.M. and Sanders, M.D., (1980) Acquired optociliary shunt vessels in papilledema. Br. J. Ophthalmol. 64: 267-271.

Frisen, L., (1982) Swelling of the optic nerve head: A staging scheme. J. Neurol. Neurosurg. Psychiatr. 45: 13-18.

Frisen, L. and Holm, M., (1977) Visual field defects associated with choroidal folds. In: Neuro-opthalmology. C.V. Mosky Co., St. Louis (Glaser, J.S., ed) Vol. XI, Chapter 19.

Galbraith, J.E.K. and Sullivan, J.H., (1973) Decompression of the perioptic meninges for relief of papilledema. Am. J. Ophthalmol. 75: 687-692. 
Galvin, R. and Sanders, M.D.. (1980) Peripheral retinal haemorrhages with papilloedema. Br. J. Ophthalmol. 64: 262-266.

Gilland. O., Tourtellotte. W.W.. O'Tauma, L. and Henderson, W.G., (1974) Normal spinal fluid pressure. J. Neurosurg. 40: 587-593.

Gowers, W.R.. (1882) A Manual and Atlas of Medical Ophthalmology. 2nd Edition, Philadelphia. Blakiston.

Green, G.J.. et al., (1980) Ischemic optic neuropathy in chronic papilledema. Arch. Opthalmol. 98: 502-504.

Griberg. T.R. and Grove. A.S.. (1980) Subretinal neovascularization and choroidal folds. Ann. Ophthalmol. 3: 245-250.

Gucer, G. and Viernstein, L., (1978) Long-term intracranial pressure recording in the management of pseudotumor cerebri. J. Neurosurg. 49: 256-263.

Guisado, R., et. al., (1975) Rebound phenomenon complicating cerebral dehydration with glycerol. J. Neurosurg. 42: 226-228.

Halpern, J.I. and Gordon. W.H. Jr., (1981) Trochlear nerve palsy as a false localizing sign. Ann. Ophthalmol. 9: 53-56.

Hart. R.G. and Carter. J.E.. (1982) Pseudotumor cerebri and facial pain. Arch. Nerol. 39: 440-441.

Hayreh. S.S., (1968) Pathogenesis of oedema of th optic disc. Doc. Opthalmol. 24: 289-411.

Hitchings. R.W., et. al. , (1976) Hemorrhages with optic nerve drusen. Arch. Neurol. 33: 675-677.

Huber, A., (1971) Eye Symptoms in Brain Tumors. C.V. Mosby Co.. St Louis, p. 104

Jamison. R.R., (1978) Subretinal neovascularization and papilledema associated with pseudotumor cerebri. Am. J. Opthalmol. 85: 78-81.

Jefferson. A. and Clark. J.. (1976) Treatment of benign intracranial hypertension by dehydrating agents with particular reference to the measurement of the blind spot area as a means of recording improvement. J. Neurol. Neurosurg. Psychiatr. 39: 627-639.

Johnston, I.H. and Paterson, A.. (1972) Benign intracranial hypertension: Aspects of diagnosis and treatment. In: The Optic Nerve. Cant, J.S., (ed), London: Henry Kingston, pp. 155-165.

Johnston, l. and Paterson. A., (1974) Benign intracranial hypertension II. CSF pressure and circulation. Brain. 97: 301-312.

Kaye, A.H., Galbraith, J.E.K. and King J., (1981) Intracranial pressure following optic nerve decompression for benign intracranial hypertension. Case Report. J. Neurosurg. 55: 453-457.

Keane, J.R. (1981) Papilledema with unusual hemorrhages. Arch Opthalmol. 99: 262-263.

Keltner, J.L., et. al., (1977) Optic nerve decompression. A clinical pathologic study. Arch. Opthalmol. 95: 97-104.

Kirkham. T.H., et. al., (1973) Unilateral papilledema in benign intracranial hypertension. Canad. J. Opthalmol. 8: 533-538.

Lorentzen, S.E., (1966) Drusen of the Optic Disc: A clinical \& genetic study. Acta Ophthalmol. Supp. 90.

Manno, N.J. et al., (1962) Intraspinal epidermoids. J. Neurosurg. 19: 754-756.

McCammon A., et al., (1981) Transient oculomotor paresis in pseudotumor cerebri. Neurology. 31: 182-184.

McCarthy, K.D. and Reed, D.J., (1974) The effect of acetazolamide and furosemide on CSF production and choroid plexus carbonic anhydrase activity. J. Pharmacol. Exp. Therapy 189: 194-201.

Merikangas, J.R. , (1978) Skew deviation in pseudotumor cerebri. Ann. Neurol. 4: 583.
Morse, P.H., et. al.. (1981) Bilateral juxtapapillary subretinal neovascularization associated with pseudotumor cerebri. Am. J. Ophthalmol. $91: 312-317$

Morris, A.T. and Sanders. M.D., (1980) Macular changes resulting from papilleodema. Br. J. Ophthalmol. 64: 211-216.

Neblett, C.R., et al.. (1972) Effect of cardiac glycosides on human CSF production. Lancet 2: 1008-1009.

Newborg, B., (1974) Pseudotumor cerebri treated by rice reduction diet. Arch. Int. Med. 133: 802-808.

Oakley, D.E. and Eltis, P.P., (1976) Glycerol and hyperosmolar nonketotic coma. Am. J. Ophthalmol. 81: 469-472.

Perlmutter, J.C., et al. . (1980) Disappearing opticociliary shunt vessels and pseudotumor cerebri. Am. J. Opthalmol. 89: 703-707.

Perret, G.E. and Graf. C.J., (1977) Subgaleal shunt for temporary ventricular decompression and subdural drainage. J. Neurosurg. 47: 590-595.

Quigley, H.A., Addicks, E.M. and Green, W.R. (1982) Optic nerve damage in human glaucoma III. Quantitative correlation of nerve fiber layer loss and visual field defect in glaucoma, ischemic neuropathy, papilledema and toxic neuropathy. Arch. Opthalmol. 100: 135-146.

Reid, A.C. and Thomson. J.A.. (1981) Absence of significant endocrine deficiencies in benign intracranial hypertension. J. Neurol. Neurosurg. Pshychiatr. 44: 731-734.

Ridsdale, L. and Moseley. I., (1978) Thoracolumbar intraspinal tumors presenting features of raised intracranial pressure. J. Neurol. Neurosurg. Pshychiatr. 41: 737-742.

Rottenberg, D.A., et. al., (1977) The effect of oral glycerol on intraventricular pressure in man. Neurology 27: 600-608.

Rush, J.A., (1980) Pseudotumor cerebri. Clinical profile and visual outcome in 63 patients. Mayo Clin. Proc. 55: 541-546.

Savino, P. and Glaser, J.. (1977) Pseudopapilledema versus papilledema. International Opthalmology Clinics. In: Clinical Neuroophthalmology: The Afferent Visual System. Vol. 17, No. I.

Shapiro. J.M., (1979) Optic topography in experimental glaucoma. Invest. Ophthalmol. 18: suppl. 166.

Snyder, D.A. and Frenkel, M., (1972) An unusual presentation of pseudotumor cerebri. Ann. Ophthalmol. 11: 1823-1827.

Spallone. A., (1981) Benign intracranial hypertension versus intracranial arteriovenous malformation. A possible CT dilemma. Acta Neurochir. (Wein) 58: 75-84.

Spence, J.D., et al., (1980) Benign intracranial hypertension without papilledema: Role of 24 hour CSF pressure monitoring in diagnosis and management. Neurosurg. 7: 326-332.

Troost, B.T., et. al., (1979) Sudden monocular visual loss in pseudotumor cerebri. Arch. Neurol. 36: 440-442.

Vander Ark, G.D., et. al., (1971) Pseudotumor cerebri treated with lumbar-peritoneal shunt. J. Am. Med. Asso. 217: 1832-1834.

Vassilouthis, J., (1979) Cerebral arteriovenous malformation with intracranial hypertension. Surg. Neurol. 41: 402-404.

Weisberg, L.A., (1975) Benign intracranial hypertension. Medicine 54: 197-207.

Wilson, D.H. and Gardner, W.J., (1966) Benign intracranial hypertension with particular reference to its occurrence in fat young women. Canad. Med. Assn. H. 95: 102-105. 